\title{
Tangence
}

\section{Marcel Proust et le nominisme : pour une philosophie proustienne du nom}

\section{Luc Benoit}

Numéro 61, décembre 1999

Savoir et littérature

URI : https://id.erudit.org/iderudit/008165ar

DOI : https://doi.org/10.7202/008165ar

Aller au sommaire du numéro

Éditeur(s)

Presses de l'Université du Québec

ISSN

0226-9554 (imprimé)

1710-0305 (numérique)

Découvrir la revue

Citer cet article

Benoit, L. (1999). Marcel Proust et le nominisme : pour une philosophie proustienne du nom. Tangence, (61), 45-72. https://doi.org/10.7202/008165ar d'utilisation que vous pouvez consulter en ligne.

https://apropos.erudit.org/fr/usagers/politique-dutilisation/ 


\title{
Marcel Proust et le nominisme : pour une philosophie proustienne du nom Luc Benoit, Universite du Québec à Montréal
}

\author{
Chascune hystoire est d'ymage illustrée \\ Affin que soit plus clairement monstrée \\ L'invention, et la rendre autenticque \\ Qu'on peult nommer lettre hierogliphicque \\ Comme jadis faisoient les anciens \\ Et entre tous les vieux Egyptiens \\ Qui denotoient vice ou vertu honneste \\ Par ung oiseau, ung poisson, une beste, \\ Ainsi ay faict, affin que l'œil choisisse \\ Vertu tant belle et delaisse le vice. \\ Gilles Corrozet, Hecatomgraphie
}

Existe-t-il une philosophie proustienne du nom ${ }^{1}$ ? En guise de réponse, nous montrerons comment certains passages de Contre Sainte-Beuve et du Temps retrouvé ${ }^{2}$ rendent compte des composantes noministes ${ }^{3}$ de la philosophie de Marcel Proust. Pour ce faire, nous examinerons la fonction poétique du nom relative aux différentes figures du temps dans l'œuvre de l'écrivain. Se rapprochant paradoxalement du concept d'involontaire proposé par Auguste Comte, l'involontaire que postule le romancier est d'origine psychosomatique ${ }^{4}$. Entre le je narrant et le je narré, le soi et

1. Cette question ne doit pas être confondue avec celle qu'adresse Vincent Descombes à propos de l'existence possible d'une philosophie proustienne du roman. Voir Vincent Descombes, Proust. Philosophie du roman, Paris, Minuit, 1987, 338 p.

2. Marcel Proust, Contre Sainte-Beuve, Paris, Gallimard, 1987, c1954, 307 p.; Le temps retrouvé, Paris, Gallimard, 1976, c1927, 442 p. Dans la présente étude, les références à ces ouvrages renvoient à ces éditions respectives.

3. "Le nominisme [...] est une façon de concevoir le langage qui privilégie la nomination comme relation sémantique par excellence. Toutes les autres catégories de signes si elles sont admises sont alors subordonnées au nom (et parfois même au nom propre) qui est vu comme le signe de base, celui à partir duquel s'édifie tout le langage. Il y a évidemment bien des variétés de nominismes possibles". Claude Panaccio, Les mots, les concepts et les choses, Montréal, Bellarmin, 1991, p. 167.

4. Lorsque nous nous référons à Auguste Comte et au positivisme, nous avons recours à l'ouvrage suivant: Auguste Comte, Cours de philosophie positive 
46

le moi, le style de l'auteur se fonde sur un rationalisme que nous qualifions de spiritualiste-relativiste. C'est précisément dans le contexte de la nomination que ce rationalisme sera abordé.

La psychosociologie de Proust converge vers l'étude des fantasmes et des illusions sensorielles liés à la représentation. Que l'on songe notamment à la synopsie qui accompagne les associations de la mémoire involontaire. Elle se rattache à la fonction poétique du nom et se module en épousant l'inversion sexuelle. En ce sens, la synopsie correspond à un dédoublement psychique. Il s'agit d'une sorte de réduplication des emboîtements théâtraux constitutifs de la personnalité humaine et se trouvant à la base des souvenirs du narrateur. Afin de décrire ce système de représentations, nous nous pencherons sur le rôle crucial du démon proustien.

La musicalité issue du nom est plus poétique que les images contenues "entre ses syllabes " pour Auguste Comte et pour Marcel Proust. Suivant la conception noministe du romancier, les noms servent de vêtements aux concepts et au personnage intérieur, tout en s'harmonisant à la musique de la petite phrase de Vinteuil. Nous montrerons comment ce leitmotiv obsessif hante les écrits de l'auteur et aboutit à la réalisation d'une idéalisation isolant "un peu de temps à l'état pur". Il importe de voir que l'écrivain exploite un point quelconque de l'espace-temps qu'il relativise à l'aide de la connotation, de l'histoire et de la fiction. Cette problématique antécopernicienne implique la recherche d'un point fondateur. Elle affecte la relation logique prévalant entre les parties et le tout que constitue $\bar{A}$ la recherche du temps perdu. La saisie de ce temps à l'état pur débouche sur un engagement ontologico-noministe.

\section{Du signe à la représentation : le nom}

L'approche dogmatique de Proust est particulière. La "proxémique" et la "phénoménologie "5 qu'il élabore fondent son jugement et la cohérence logique de la coexistence spatio-temporelle

(Première et deuxième leçons). Discours sur l'esprit positif, 2 vol., Paris, Garnier Frères, 1949. L'étude de Lucien Lévy-Bruhl, La philosophie d'Auguste Comte (Paris, Félix Alcan, 1913, 416 p.) étaye également notre propos.

5. Il va de soi que les concepts entre guillemets sont approximatifs et que Proust ne connaissait probablement ni la phénoménologie d'Edmund Husserl, ni la proxémique. En revanche, il avait certainement entendu parler du phénomé- 
des mots ou des choses. Elles lui permettent de construire un récit jouant adéquatement sur l'illusion référentielle. Ce procédé crée un effet de style aux allures psychédéliques ${ }^{6}$. Voilà en premier lieu comment le processus scriptural de Proust pourrait instaurer des relations sémantiques entre l'idéalisation artistique et l'abstraction propre à l'activité intellectuelle des scientifiques.

$\mathrm{Au}$ niveau social, l'écrivain entend bien situer sa différence intellectuelle au sommet de la pyramide littéraire ${ }^{7}$. Rappelons que vers la fin du $\mathrm{XIX}^{\mathrm{e}}$ siècle, les intellectuels commencent à former une nouvelle classe sociale qui se mêle au tout Paris et fréquente les gens du monde. Pour Proust, il importe peu que la différence des classes sociales soit réelle ou imaginaire. L'essentiel, c'est que les personnages du roman partagent cette croyance populaire. Qu'ils y croient ou qu'ils feignent d'y croire, l'effet demeure sensiblement le même dans l'optique du romancier. En d'autres termes, bien que la différence s'applique de façon homogène à l'espèce, elle la transcende pour atteindre le genre. Dans le cas présent, le genre serait "animal littéraire" et l'espèce, "mondaine». Si Proust entre dans l'espèce mondaine, sa différence individuelle la déborde accidentellement. Il faut comprendre qu'un accident, tel que l'inversion sexuelle par exemple, "admet le plus et le moins", contrairement à la différence qui n'est aucunement soumise à un "degré d'intensité" 8 .

Au sein de la Recherche, l'approche noministe a pour fonction première d'établir une rupture analytique entre la représentation, l'idéalisation et l'abstraction?'. Les prémisses méthodo-

nisme d'Ernst Mach. Notons que le romancier s'intéressait aussi aux découvertes de Bergson et d'Einstein.

6. L'étude d'Antoine Compagnon demeure l'une des plus instructives en ce qui a trait à l'onomastique proustienne. Voir Antoine Compagnon, Proust entre deux siècles, Paris, Seuil, 1989, p. 229-256.

7. La différence est un des cinq prédicables attribués à Porphyre par Guillaume d'Ockham dans son Commentaire sur le livre des prédicables de Porphyre précédé du proême du commentaire sur les livres de l'art logique. Introduction et présentation de Louis Valke, traduction de Roland Galibois, Sherbrooke, Centre d'études de la Renaissance, 1978, 212 p. Guillaume d'Ockham (ou Occam) serait né vers la fin du XIII ${ }^{\mathrm{e}}$ siècle en Angleterre. Théologien et philosophe, il était aussi sémiologue. Il demeure l'un des précurseurs importants de l'empirisme de Hume et de la sémiotique de Locke.

8. Guillaume d'Ockham. op. cit., p. 196-197.

9. On ne saurait trop insister sur l'étude de Joël Biard, notamment la partie présentée depuis le cinquième jusqu'au dixième chapitre. Voir Joël Biard, 
logiques sur lesquelles se fondent cette assertion sont hypothétiques. Nous pensons que le nominisme de Proust s'apparente sous plus d'un angle au nominalisme de Guillaume d'Ockham ${ }^{10}$. C'est pourquoi nous postulons que la philosophie proustienne du nom et son traitement esthétique demeurent dans le voisinage du nominalisme développé par Ockham au xIv ${ }^{\mathrm{e}}$ siècle $^{11}$.

Le philosophe médiéval montre clairement qu'il n'y point d'universaux extramentaux. Seuls les individus existent. Par exemple, l'humanité diffère de Proust, mais elle ne se distingue pas des hommes en général et de leur série. L'humanité est réductible à la somme des particuliers; sinon, il y a une appréhension évasive du réel, ou pire encore, une régression infinie et, par conséquent, une inconsistance d'ordre logique. Pour sa part, l'écrivain circonscrit la signification du nom "humanité" à l'intérieur de l'espèce mondaine et réduit cette dernière à la série d'individus qui la composent. L' "humanité" au sens strict se limite à la société nobiliaire et à la parenté immédiate. En un sens large, "humanité" désigne aussi les individus du demi-monde. Il s'agit des bourgeois. C'est ainsi que le romancier conçoit l'être sur un plan social. Cependant, l'être apparaît dans le néant et c'est là que se positionnent le plus grand nombre d'individus. Selon Proust, ces gens s'apparentent au néant, c'est-à-dire une sorte de masse obscure informe et anonyme. Ces personnes atteignent l'éternité par la mort tandis que le grand écrivain y touche par la création d'une ouvre littéraire. Pour Proust et Ockham, la généralisation s'inscrit à l'intérieur d'un rapport de signification qui a trait à la constitution du signe et à la série qui le sous-tend. Le signe est l'unité abstraite d'une série d'individus réels ou possibles. Ces entités se subsument dans l'universalité du nom commun et en constituent la signification.

Ockham rompt avec la métaphysique et l'ontologie traditionnelles dans la mesure où il limite la signification de l'humanité en dénombrant les individus qui la composent. Le signe s'érige en fonction de son intelligibilité à l'intérieur de la mémoire et de la

Guillaume d'Ockham. Somme de logique. Première partie, Mauvezin, T.E.R., 1988 , p. $16-39$.

10. Afin de clarifier la pensée de Guillaume d'Ockham, nous nous référons plus particulièrement à l'étude de Pierre Alféri: Guillaume d'Ockham. Le singulier, Paris, Minuit, 1989, 489 p.

11. Antoine Compagnon établit un rapprochement similaire entre Ockham et Montaigne dans Nous, Michel de Montaigne, Paris, Seuil, 1980, 232 p. 
phrase. Graduellement, il s'abstrait de la représentation. Le penseur médiéval accorde aux illusions procédant de la représentation la place qu'elles méritent, c'est-à-dire celle qu'elles doivent occuper en tant que phénomène résiduel et de second ordre par rapport à l'intellection. Proust se joue aussi des illusions sensorielles liées à la représentation. Dans ce contexte, l'approche du romancier est davantage leibnizienne puisqu'il décrit le particulier en le réduisant et en le mettant en abîme au cour du reflet de l'Univers. Aussi le personnage intérieur est-il à l'image d'une monade. Quant à Ockham, il considère l'existence sous l'angle de la pratique linguistique et de l'étude des actions. Compte tenu de cette insistance sur les actions, l'existence perd son caractère trivial et traditionnel d'ontologie spéculative. Célèbre commentateur de la philosophie ockhamienne, Pierre Alféri assigne à l'existence un rôle non négligeable. Précisons que l'approche d'Alféri ne relève pas exclusivement du nominalisme. Elle s'oriente plutôt vers une phénoménologie nominaliste:

En outre, l'existence est ici un simple paramètre, positif ou négatif dans l'intuition, annulé dans l'abstraction. Existant ou non-existant: le "Ou" est exclusif dans l'intuition, la différence s'y marque avec évidence; le "ou" est inclusif dans l'abstraction, la question de l'existence est indifférente, ne se pose pas et le paramètre s'annule. C'est bien comme simple paramètre que l'existence est l'indice de l'intuition. ${ }^{12}$

À la lumière de ces considérations, on peut constater que le singulier incarne une "essence-existence" justifiée par la pratique discursive et l'analyse logique des actions. L'intuition et l'abstraction permettent de reconstituer la chaîne sérielle des possibles répertoriés sous le signe. Or, l'intuition est fondatrice et plus originaire que l'abstraction. C'est une évidence judicative car il importe d'abord d'appréhender un particulier avant de procéder à une généralisation. L'intuition se pose donc comme nécessaire afin de remonter la chaîne causale que présuppose l'habitus. Quant à l'abstraction, elle demeure relative au temps vécu et factuel: "La séquence intuition-abstraction, vue de la choseévocation de la chose, qui est l'étalon ou le module du temps vécu, la source de la conscience intime, est marquée par une irréversibilité de fait. " ${ }^{13}$ Dans l'optique proustienne, les sensations de

12. Pierre Alféri, op. cit., p.160.

13. Ibid., p. 196. 
50

temps coexistent et accompagnent toutes les sensations de la mémoire. Le passé et le présent se distinguent parce que la séquence intuition-abstraction est irréversible. Considérée par le romancier comme une catégorie de l'esprit, la durée s'insère dans les constituantes du souvenir; elle est sous-entendue derrière chaque élément phrastique, chaque raisonnement, chaque monde possible. Au fil du temps et de l'expérience, le langage des signes s'intellectualise en passant de l'involontaire au volontaire, du féminin au masculin, puis de Gomorrhe à Sodome, du temps perdu au "temps retrouvé". Ainsi, le temps de la Recherche procède d'une "colligation ${ }^{14}$ " générée par la substance de l'être. La distorsion schématique et musicale de l'être-temps est le fondement philosophique du roman proustien.

Le rôle de l'imagination est déterminant pour Proust puisque cette faculté permet d'éviter les automatismes involontaires du langage qui détruisent le beau style. Le but du romancier est de créer une impression stylistique durable ancrée à une intuition esthétique originaire. C'est un mécanisme rétroactif lié au continuum mnésique qu'Alféri décrit en ces termes: "Dans cette genèse de l'abstraction, nous avons d'un seul coup traversé plusieurs étapes: abstraction première accompagnant toute intuition, habitus abstractif comme cause interne de la répétition, abstraction maintenue seule dans la répétition, ou souvenir proche désormais libéré de l'intuition. "15 Au sein de la poétique proustienne, ce mécanisme a surtout trait à l'inversion sexuelle, à la durée et aux sensations de temps ${ }^{16}$. On peut décrire ce mécanisme de la façon suivante: l'impression première est pleine de scories; elle ne participe ni de la représentation volontaire ni de l'habitus; elle est cinesthésique et affectée à la collection intuitive des données éparses; la prise de conscience ultime se réalise uniquement au souvenir proche. Lors de la rédaction de son ouvrage romanesque, Proust tend à ne conserver que l'intuition originaire et le souvenir proche, même s'il doit pour cela occulter de nombreuses sources en les pastichant. Il subordonne

14. Husserl emploie ce terme pour décrire une connaissance doxique ensembliste identitaire qui précède l'expérience. Voir Edmund Husserl, Expérience et jugement, traduit de l'allemand, Paris, Presses universitaires de France, 1991.

15. Pierre Alféri, op. cit., p. 197.

16. Voir Félix Lacher dit Ravaisson-Mollien, De l'habitude, Tours, Fayard, 1984, c 1838 , p. 367. 
les citations de toutes provenances à l'inspiration que lui procure son démon intérieur. Tout bien considéré, l'écrivain offre une interprétation originale du "monde" en rapport étroit avec le "kaléidoscope social" de Schopenhauer ${ }^{17}$.

S'il y a un "temps à l'état pur" qui est synonyme d'éternité dans la Recherche, il apparaît dans un firmament constellé de métaphores au sein desquelles le reflet de la voûte céleste est mis en abîme. Ce phénomène rappelle le parcours étoilé de Leibniz. Précisons à l'instar du mathématicien William R. Hamilton que, sur un parcours en forme d'étoile, la distance périphérique est toujours égale par rapport au centre. Proust suggère qu'il n'y a pas de chemin privilégié pour atteindre le cœur de l'ineffable et de l'innommable. Il s'interroge quant au sens de l'existence et du langage en les plaçant entre parenthèses. De ce fait, il attire l'attention du lecteur sur l'inconscient. Quant au démon intérieur, son rôle est celui d'un encyclopédiste ayant pour mission d'inspirer le particulier en se nourrissant exclusivement d'idées universelles. À la manière d'un Emmanuel Kant, cette entité tente de subsumer le particulier dans l'universel, mais elle est consciente de sa situation de prisonnière à l'intérieur du corps qu'elle habite ${ }^{18}$. Ce paradoxe impliquant l'universel et le particulier ne se dénoue que dans $L e$ temps retrouvé. À ce moment, la figure auctorielle, comparable à un vecteur ou à un tenseur, converge vers celle du narrateur et du héros. Autrement dit, l'emboîtement infini des théâtres de l'intériorité rejoint le théâtre fini de la réalité. La réduplication des habits d'Arlequin s'intègre à l'automate fini de la réalité ${ }^{19}$.

Suivant la philosophie que Proust élabore, la genèse mémorielle de l'abstraction produite par l'intuition et la répétition se confond avec la constitution du temps vécu. Elle se libère de l'image afin qu'on puisse retrouver le temps au souvenir proche. À ce chapitre, il importe de souligner qu'Alféri conçoit l'habitus comme une abstraction libre qui ne dépend plus de la perception des choses matérielles: "Ce n'est plus quelque chose hors de moi, c'est quelque chose en moi qui désormais motive mes

17. Voir Arthur Schopenhauer, Le monde comme volonté et comme représentation [1818], Paris, Presses universitaires de France, 1966, 1434 p.

18. De là découle peut-être en partie l'idée de "la prisonnière" qu'il est possible de mettre en relation avec le réalisme des universaux.

19. Leibniz établit cette distinction originale entre le monde réel de l'automate fini et les mondes possibles de l'automate infini. 
52

actes ${ }^{20}$. "Ce principe d'apprentissage par répétition doit être considéré comme une phase transitoire, une cause efficiente. Les automatismes psychiques sont à la base de toutes les variations qu'impose l'abstraction à l'objet singulier. Cette activité est comparable au réglage minutieux de la texture d'un caractère d'impression: "Grâce à l'habitus et la répétition, c'est une libération de la trace que l'abstraction accomplit dans son exercice concret. " 21 Partant du souvenir proche, Proust considère la reconstruction d'une réalité en l'envisageant successivement sous de multiples angles et quatre dimensions. L'imagination se dégage de l'habitus grâce à l'analogie, aux métaphores et aux associations involontaires qui sous-tendent la relativisation du souvenir. Parallèlement aux continuums quadridimensionnels du temps et de la mémoire, le pastiche volontaire de l'écrivain aide à penser la durée et les facettes cachées d'un objet.

De façon idéale pour le romancier, l'énumération-nomination ne doit jamais être éclipsée par la répétition ou certaines dispositions psychiques qui ont le caractère d'un habitus. Quant à l'activité de l'intellect, Ockham la distingue de la représentation en montrant qu'elle se rapporte toujours à une essence singulière. Aux dires de l'écrivain, l'intelligence servirait à ordonner la collection des souvenirs de façon à pourvoir le texte d'une invariance stylistique. Selon un point de vue opposé, celui des scientifiques positivistes, l'intellection précède l'imagination. L'activité de l'intellect ne revêt guère la même importance pour les artistes et les positivistes. Pour les premiers, elle ne constitue qu'un moyen, non une fin en soi. L'inversion de l'ordre intellect-imagination provient du concept. Alféri décrit ainsi ce phénomène: "En particulier, ils semblent permettre une inversion de l'ordre intuition-abstraction: grâce au concept d' "homme", je peux penser à un homme que l'on m'a désigné par ce concept, comme "l'homme qui a gagné la bataille d'Austerlitz", avant de l'avoir vu. " ${ }^{22}$ Il est donc possible de raisonner à partir de l'abstraction en focalisant l'attention sur une donnée imaginaire. Force est de constater que l'approche proustienne des illusions perdues est comparable à celle d'Ockham. Lorsque ce dernier lit Pierre d'Auriole, il note que les illusions ne diffèrent pas des sensations ordinaires et sont difficiles

20. Pierre Alféri, op. cit., p. 197.

21. Ibid., p. 197.

22. Ibid., p. 211. 
à cerner. Ockham tente à juste titre de les évacuer progressivement de sa philosophie. Il fait du concept un interprétant tendant à se dégager des représentations, des images, des hallucinations.

Ockham et Proust ont ceci en commun: ils considèrent que le concept est l'objet qu'il faut juger à l'intérieur des procès d'intention. Comme le décrit Alféri, l'opposition ockhamienne entre la représentation et l'intellection ressemble à celle proposée par l'œuvre proustienne. Selon d'Auriole, ce qu'on soutient au sujet de la sensation, on doit le maintenir également pour l'imagination et le sens interne. Lorsque l'on imagine sa mère par exemple, on l'appréhende comme un être intentionnel qui est semblable ou différent de son être réel. Ockham explique comment l'amour est impliqué dans un conflit des facultés se divisant en un acte appréhensif et judicatif. Au fil de la Recherche, Proust montre que l'amour échappe aux volitions et à l'intentionnalité. Son caractère involontaire pourrait dépendre de la neurasthénie. L'amour est posé comme une maladie incurable. À ce niveau, il faut admettre que la politique positiviste de Comte ainsi que les réflexions d'Ockham et de Proust s'harmonisent avec brio:

[...] l'acte d'aimer est naturellement antérieur à cet acte [de savoir] et cet acte de savoir est simultané à l'acte d'aimer. Mais l'acte d'aimer présuppose la notitia incomplexe de Sortes, ou quelque autre, et il est simultané à celle-ci. Donc ces trois actes sont simultanés, les deux [extrêmes] dans l'intellect, celui du milieu dans la volonté. ${ }^{23}$

La mise en rapport de ces systèmes de pensée prend appui sur une conception bien particulière du réalisme. Mentionnons que Guillaume d'Ockham s'évertue à critiquer le réalisme philosophique de Duns Scot. D'où la distinction établie entre le nominalisme et le réalisme au sein de ses écrits:

[...] on est nominaliste aussi longtemps que l'on suppose qu'un concept signifie une structure, on devient réaliste au moment où, dans la démarche réductive, on suppose que les concepts signifient, non des structures, mais des natures structurantes, des principes de structuration. ${ }^{24}$

23. Voir Guillaume d'Ockham. Prologue du commentaire des Sentences, question $I$ (extrait), A. de Muralt, "La connaissance intuitive du néant et l'évidence intuitive du je pense", Studia philosophica, 36, Bâle, 1976, p. 112.

24. Nous reproduisons intégralement la citation tirée du texte de Guillaume d'Ockham, Commentaire sur le livre des prédicables de Porphyre précédé du proême du commentaire sur les livres de l'art logique, p. 23. 
54

Pour les raisons que nous venons d'évoquer, nous soutenons qu'Ockham est un nominaliste radical. En ce sens, le nominisme de Proust offre de multiples analogies avec le nominalisme du philosophe, sans compter que le romancier mentionne de façon explicite le nom de Duns Scot à l'intérieur de Jean Santeuil ${ }^{25}$. À la polémique opposant le nominalisme au réalisme se greffe celle du fictum et de l'intellection. Il s'agit d'une façon de philosopher à partir d'une représentation ou des signes. À la suite d'Ockham, Marcel Proust se tourne davantage vers les signes. L'écrivain envisage l'expression et la représentation sous un angle projectif s'apparentant de près à la théorie des ombres de Leibniz. Selon le romancier, il existe différents degrés d'abstraction dans un espace des paradoxes régi par une logique du faux. Similaire à la vérité décrite par Leibniz, la vérité proustienne comporte une certaine opacité.

Dans le but de cerner la philosophie proustienne du nom constituée à partir des signes, reportons-nous de nouveau à Alféri et, plus précisément, à son commentaire sur le signe. Ce dernier postule que le concept est un signe: "La théorie du fictum serait comme un échafaudage qu'il faut faire tomber une fois l'édifice achevé." ${ }^{26}$ Le philosophe se méfie du fictum-représentation parce qu'il ne peut expliquer, ni aider à comprendre le lien qu'entretient le langage avec la référence. La représentation n'autorise pas la désignation en ligne droite d'un étant, ici, maintenant. Elle entretient le cercle vicieux de la dérivation infinie et illusoire. À l'inverse de la représentation, le concept se pose tel un déictique désignant une série de singuliers en ligne droite. Alféri distingue la désignation in recto des cas in obliquo qui s'orientent vers les aspects sensibles et les qualités générales de l'objet. La connotation in fictum procède de l'imagination et de la représentation. Elle est le lieu de l'épithète et des tropes. Les concepts essentiels renvoient précisément à l'objet extérieur considéré de facto, ou en imagination. Alféri montre pourquoi le signe conceptuel est naturel:

Il est donc définitivement établi que le cour du concept est le pur mouvement de la référence, en un acte indérivable. Ce

25. Cette hypothèse semble raisonnable. Dans Jean Santeuil, se trouve un long passage faisant état de la confusion phonétique qu'engendre le rapprochement du nom de Duns Scot avec celui de Walter Scott. Voir Marcel Proust, Jean Santeuil, édition établie par Pierre Clarac avec la collab. d'Yves Sandre, Paris, Gallimard, 1971, p. 408.

26. Pierre Alféri, op. cit., p. 229. 
mouvement est aveugle, irréductible à une représentation, il ne peut, à la rigueur, que s'orienter selon une image dans certains concepts obliques et connotatifs. Le concept est bien un signe s'inscrivant naturellement dans l'esprit. ${ }^{27}$

Au sein de son œuvre romanesque, Proust évoque comment le signe est naturel et involontaire chez l'amoureux, le neurasthénique, l'homosexuel. Il insiste à sa manière sur l'aspect intuitif et naturel du signe. Selon l'écrivain, le statut du signe est ambigu lorsqu'il s'applique au domaine de la sexualité. Par exemple, quand le signe indique la présence de l'androgyne, il est difficile d'établir avec certitude s'il est toujours conscient. Suivant la conception proustienne qui se donne à lire au cour des textes, les signes subjectifs sont à divers degrés plus réels que les signes matériels. L'auteur de la Recherche accorde autant de réalité au signifié qu'au signifiant.

Dans un passage du Temps retrouvé, le romancier s'oppose diamétralement à la littérature réaliste. Son argumentation repose sur un nominisme dérivé du nominalisme. À l'exemple d'Ockham, il organise ses idées et les "contextualise" en rapport avec le temps vécu. L'extrait suivant témoigne de façon magistrale du plaidoyer anti-réaliste de Proust:

Tel nom lu dans un livre autrefois contient entre ses syllabes le vent rapide et le soleil brillant qu'il faisait quand nous le lisions. De sorte que la littérature qui se contente de "décrire les choses ", d'en donner seulement un misérable relevé de lignes et de surfaces, est celle qui, tout en s'appelant réaliste, est la plus éloignée de la réalité, celle qui nous appauvrit et nous attriste le plus, car elle coupe brusquement toute communication de notre moi présent avec le passé, dont les choses gardaient l'essence, et l'avenir, où elles nous incitent à la goûter de nouveau. C'est elle que l'art digne de ce nom doit exprimer, et, s'il y échoue, on peut encore tirer de son impuissance un enseignement (tandis qu'on n'en tire aucun des réussites du réalisme), à savoir que cette essence est en partie subjective et incommunicable. ${ }^{28}$

Conformément à l'activité d'écriture proustienne, Ockham s'attarde aux relevés de lignes et de surfaces des réalistes ${ }^{29}$. Il

27. Ibid., p. 253.

28. Marcel Proust, Le temps retrouvé, p. 244-245.

29. Somme de logique, p. 152-159. 
56

affirme que la phrase est une "quantité discrète" qui entre accidentellement dans le genre de la quantité, au même titre que les termes lieu et temps: "[...] le lieu est un prédicable qui ne se distingue pas de la ligne, de la surface et du corps, de la même manière que ces derniers se distinguent entre eux. " 30 Le philosophe juge arbitraire la répartition des unités distinctes lorsqu'elle ne tient pas compte du "tout" ou du contexte auquel elle se rapporte.

Proust élabore une esthétique nominale en combinant différentes sémiologies. Comme nous l'avons mentionné, l'écrivain utilise les noms en tant que figures du temps. En fait, faut-il préciser que le temps abolit plus facilement l'existence des individus et des choses que leurs noms. Les commentaires d'Alféri portant sur l'expérience qui creuse la distance spatio-temporelle entre la référence et la deixis sont révélateurs du nominisme à l'œuvre chez Proust. Alféri note que "[la] référence est seulement l'analogue d'une deixis, d'une monstration des existences présentes, passées et possibles "31. L'intuition et la référence prolongent l'effacement progressif de la deixis, tandis que l'abstraction la neutralise. L'intuition et l'abstraction entrent dans l'horizon et la complexité croissante du jeu de la deixis en montrant du doigt des objets mis en commun. Nous pouvons dès lors affirmer que "[...] tout signe commun est comme une collection de noms propres" 32 au sein des textes proustiens étudiés.

\section{Le cygne-signe et l'étang-étant : universalité et particularité du nom Guermantes}

Selon Ockham, le signe se construit en fonction des signifiés extérieurs. La signification est alors décrite comme une relation irréelle établie entre les choses réelles que sont les signes et les individus qu'ils dénotent dans le monde. Partant de cette idée, Alféri montre que le concept est déjà un signe avant même d'être nommé: "Il vaudrait mieux dire, dans le langage de Peirce, que le concept est un signe interprétant à l'égard du signe verbal. "33 Le concept demeure un signifiant autonome recouvert par

\footnotetext{
30. Ibid., p. 154.

31. Pierre Alféri, op. cit., p. 272.

32. Ibid., p. 273.

33. Ibid., p. 280.
} 
d'autres signifiants, c'est-à-dire les noms formulés à l'oral ou à l'écrit. Le nom ne signifie pas le concept; il est un signifiant subordonné à celui-ci. Les noms et les concepts appartiennent à différents systèmes langagiers qui sont parallèles. Le langage naturel et le signe indiciel ne permettent pas d'établir la vérité ou la fausseté d'une proposition. La dimension judicative de la pensée et les signes arbitraires de la langue sont complémentaires au signe naturel. On pourrait supposer que le signe naturel-indiciel est involontaire parce qu'il s'inscrit causalement dans l'esprit ou par accointance, comme pourrait l'affirmer Bertrand Russell. Pardelà l'intellection, les concepts font partie d'un langage mental et naturel ${ }^{34}$. Les mots écrits ou oraux résultent d'un langage institutionnalisé et conventionnel. Au Temps retrouvé, Proust présume que les intermittences du cœur et du personnage intérieur sèment la confusion dans l'esprit des amoureux. Il tient alors un discours sur le caractère imprécis du langage de l'amour-propre:

Or si, quand il s'agit du langage inexact de l'amour-propre par exemple, le redressement de l'oblique discours intérieur (qui va s'éloignant de plus en plus de l'impression première et centrale) jusqu'à ce qu'il se confonde avec la droite qui aurait dû partir de l'impression, si ce redressement est chose malaisée contre quoi boude notre paresse, il est d'autres cas, celui où il s'agit de l'amour par exemple, où ce même redressement devient douloureux. 35

Pourquoi le langage de l'amour-propre est-il approximatif? Parce qu'il est connotatif et oblique. En principe, c'est une droite qui aurait dû partir de "l'impression première et centrale". Le romancier affirme que le discours oblique se rapporte à la mémoire involontaire, à l'amour, à la représentation poétique du monde dont fait état son livre intérieur. À bien des égards, ce livre est la traduction romanesque du langage mental ${ }^{36}$. Voilà manifestement un savoir nominaliste à l'œuvre dans la Recherche.

34. Pour en savoir davantage sur certaines circularités de raisonnement et quelques inconsistances logiques chez Ockham, il convient de consulter Cyrille Michon: Nominalisme. La théorie de la signification d'Occam, Paris, Vrin, 1994, $525 \mathrm{p}$.

35. Marcel Proust, Le temps retrouvé, p. 251.

36. Il ne s'agit évidemment que d'une analogie. Cependant, chez Proust, cette analogie va encore plus loin, comme si les interactions possibles entre le langage naturel ou conceptuel et le langage conventionnel pouvaient donner lieu à la création de différentes géométries. 
58

Pour décrire la fonction de nomination qui caractérise la poétique proustienne, il importe de se reporter au vocabulaire de la logique. Il s'agit d'abord des mots dotés d'une existence autonome. Ces mots sont appelés catégorèmes. Puis, il y a d'autres mots qui ne possèdent pas d'existence autonome. Ce sont les syncatégorèmes. Pensons à "tous", "aucun", "en outre". Les catégorèmes sont autosémantiques et les syncatégorèmes, synsémantiques, suivant leur degré d'autonomie à l'intérieur de l'enchaînement syntaxique de la phrase. Les syncatégorèmes servent à modifier et à compléter les catégorèmes ${ }^{37}$. Dans le but d'immortaliser ses souvenirs, Proust élabore une dynamique phrastique. Il s'inspire de la durée einsteinienne et du schéma comtien de l'expression. Il exploite la représentation du train telle une métaphore exprimant la vitesse relative de la lecture. Nous supposons alors que, dans ce cas précis, les catégorèmes sont illustrés comme s'il s'agissait de locomotives, tandis que les syncatégorèmes représentent des wagons. À un autre degré d'abstraction (Ockham dirait en supposition matérielle), ce sont les syllabes qui se métamorphosent en locomotives. Le rythme de la lecture s'apparente à la vitesse du train et les rails aux modalités poétiques d'Aristote, c'est-à-dire: le possible, l'impossible, le nécessaire, le contingent. Les phrases du livre intérieur sont filtrées par ces modalités. La sprezzatura proustienne implique une chaîne de nécessités qui soustrait le style aux contingences du temps grâce à l'emploi involontaire des métaphores. Le style involontaire de Proust, c'est la sprezzatura de l'esthétique décadente. Les mondes possibles s'intègrent aux "anneaux métaphoriques du beau style", tels un mandala, les roues de Lulle, une vision télescopique et sublime des paysages, des décors, des scènes érotiques. Ce jeu analogique tisse des liens avec le relativisme d'Einstein ou, plus précisément, suggère un parallèle entre l'espace-temps à quatre dimensions de Minkowski et "la psychologie dans le temps" de Proust, c'est-à-dire l'étude d'une durée psychique, connotée de façon encyclopédique. On comprend alors pourquoi l'écrivain insiste tant sur le contexte et la problématique de la référence.

Si l'on veut bien saisir le processus de la référence à l'intérieur des textes proustiens, il importe de s'attarder à la théorie

37. Voir Guillaume d'Ockham, Sur l'Universel: Commentaires sur les sentences, livre I, dist. II, quest. 8, traduction de H. Poitevin, Philosophie, 30, 1991, p. 26-27. 
des suppositions développée par Ockham. Ce dernier a construit une théorie des "suppositions" dont il distingue principalement trois types permettant d'observer la référence en acte et en puissance $^{38}$. Il s'agit de la "supposition personnelle", de la "supposition matérielle", de la "supposition simple". En "supposition personnelle", les signes tiennent lieu ou supposent pour leurs référents en tant que déixis, ce qui constitue une illusion référentielle. La supposition personnelle se subdivise en huit autres suppositions ${ }^{39}$ :

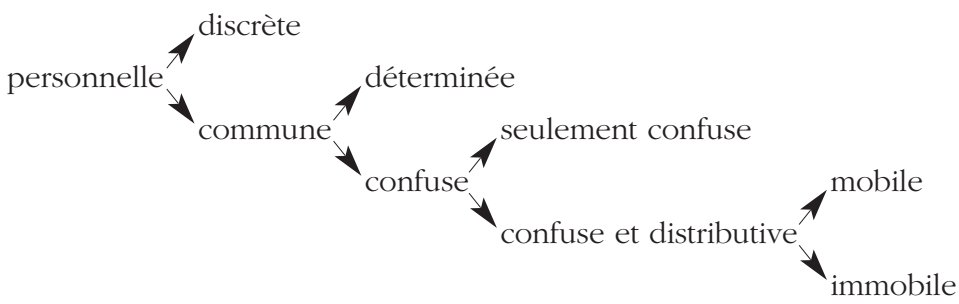

En "supposition matérielle" et "simple", les signes demeurent eux-mêmes. Dans le premier cas, ce sont des signes matériels, et dans le second, des signes mentaux ${ }^{40}$. En général, les signes indiquent le référent plus ou moins déterminé en supposition personnelle. L'emploi impropre des signes mène à l'étude des tropes. Alféri en analyse quelques-uns au nombre desquels figurent la métaphore, la métonymie, la synecdoque, l'antonomase. Il montre comment les tropes s'apparentent à "un jeu dans la référence, où le signe fait référence aux étants qui seraient nommés en propre par un autre signe " ${ }^{41}$. L'auteur précise que les références impropres et équivoques "ne sont, respectivement, que le remplacement d'une référence par une autre et le couplage de deux références irréductibles l'une à l'autre " 42 .

Dans la Recherche, le romancier divise le parcours initiatique de son personnage principal en trois niveaux: l'âge de l'enfance qui est lié à la poésie des noms, l'âge de mots correspondant au moment où le héros perd ses illusions sur la vie, puis l'âge

38. Pierre Alféri, op. cit., p. 300-308.

39. Voir Joël Biard, Guillaume d'Ockham, Logique et philosophie, Paris, Presses universitaires de France, 1997, p. 49.

40. Nous simplifions à l'extrême la théorie de la supposition d'Ockham.

41. Pierre Alféri, op. cit., p. 329.

42. Ibid., p. 331. 
60

adulte, qui est plus positif et davantage associé aux choses, aux préoccupations matérielles de ce monde ${ }^{43}$. Globalement, ce découpage rend compte de la désillusion résultant de la maturation intellectuelle du héros dans le récit. Lorsqu'il s'agit de cerner la noblesse poétique du nom, l'analyse de la nomination en fonction de la série et de la connotation s'avère des plus pertinentes. Proust procède à cette opération intellectuelle dans Contre Sainte-Beuve. Il s'intéresse à l'universalité du nom Guermantes. Il débute en précisant qu'il désire se libérer des automatismes langagiers. Il envisage son étude des noms dans une perspective proprement littéraire. Le nom de madame de Guermantes évoque la noblesse du temps présent et du xiII ${ }^{\mathrm{e}}$ siècle. L'écrivain compare alors les divers Guermantes à une pierre aristocratique. La métaphore s'avère juste dans la mesure où le cour des aristocrates est dur comme de la pierre. La pierre nominale Guermantes s'intègre à l'architecture de la Recherche, ainsi qu'à une interprétation positiviste et raisonnée du monde. Cette interprétation positiviste du monde s'achève par des réflexions noministes savantes concernant les différentes fonctions sémiologiques du langage.

Proust met l'accent sur l'appréhension de l'objet observé et nommé auquel il attribue une signification toute particulière ${ }^{44}$. La série considérée devient alors métaphorique. Elle engendre un effet poétique d'éloignement qui s'harmonise aux présumées associations involontaires de la mémoire. L'image du train illustre la série Guermantes à l'intérieur d'un récit spatio-temporel et mémoriel. Cette série se rapporte logiquement et concrètement à son domaine, c'est-à-dire au vieux château des Guermantes. Le nom Guermantes et le château surgissent parfois à gauche ou à droite du chemin de fer. L'intégration subjective de la série s'effectue à partir d'un retournement du point horizon à l'intérieur de la mémoire. Ce chiasme implique la réduplication de multiples dimensions qui croisent l'infiniment grand et l'infiniment petit. Il oriente asymptotiquement la conscience vers la focalisation zéro

43. Voir Luc Fraisse, Proust au miroir de sa correspondance, Liège, Sedes, 1996, p. 41.

44. Selon Comte, la parole est née du chant, et dans le règne animal, toute la représentation se réduit à une mimique plus ou moins expressive. C'est aussi valable pour les arts qui imitent et idéalisent à différents degrés. Voir Auguste Comte, Système de politique positive, tome 1, Paris, L. Mathias, 1851, p. $288-289$. 
du regard. Proust contemple l'espace-temps auquel se rapportent la série Guermantes et leurs domaines. Il va sans dire que la distinction entre la gauche et la droite ne revêt plus d'importance, puisqu'elle est relative au positionnement de l'observateur. L'écrivain considère le monde extérieur comme s'il s'agissait tour à tour d'un miroir simple, concave, ou convexe. Pour le romancier, le réalisme scientifique de l'époque procède toujours d'un plan, d'une donnée classique presque comparable au mouvement mécanique des escaliers roulants qui caractérisent la rigueur du style de Gustave Flaubert. Contrairement à Flaubert et aux positivistes, Proust tente d'apporter un peu de relief poétique et pittoresque au style de ses écrits. Pour ce faire, il relativise la psychologie plane de son époque en employant métaphores et topiques. Il fragmente les différentes composantes topologiques du discours en toponymes et en topiques. La métaphore introduit des éléments paradoxaux et originaux à travers ces lieux communs. Elle permet de jouer sur les transpositions nominales et analogiques des références à la manière dont l'artiste peintre dispose les couleurs sur un tableau. Il s'agit presque d'un nominalisme pictural.

Dans Contre Sainte-Beuve, l'écrivain réitère la représentation de la locomotive. Il prétend que les noms nobles sont charmants parce qu'ils désignent souvent des châteaux ou des stations de chemin de fer. Par exemple, le nom Guermantes dénote des lieux historiques. Les noms propres ont une fonction dénotative et ils précèdent les objets qu'ils désignent, snobisme oblige. Le chemin de fer connote le style des Guermantes et le train est une métaphore de cette lignée aristocratique. Plus précisément, la voie ferrée illustre la cohérence stylistique connotant cette série de particuliers. Les différentes séries sont comparables. Les éléments appartenant à l'une d'entre elles peuvent connoter analogiquement les particuliers d'une autre. Plusieurs séries sont enchevêtrées par la connotation, telles des voies ferrées qui se croisent. Le romancier utilise le nom comme un instrument d'optique qui dénote un point particulier de sa mémoire et un espace-temps concomitant avec une région de son registre encyclopédique. L'analyse topologique de l'imaginaire proustien se précise peu à peu: "C'est encore aujourd'hui un des grands charmes des familles nobles qu'elles semblent situées dans un coin de terre particulier, que leur nom qui est toujours un nom de lieu, ou que le nom de leur château (et c'est encore quelques fois le même) 
62

donne tout de suite à l'imagination l'impression de la résidence et le plaisir du voyage. " 45

Lorsqu'il se dirige vers le château Guermantes, le narrateur comble un espace physique. Au niveau psychologique et existentiel, la distance qualitative entre le romancier et le domaine peut varier à l'infini. Cette distance est toujours connotée de façon encyclopédique. À la limite, elle pourrait changer infiniment en passant de la sphère de l'ontique à celle de l'ontologie qui s'éloigne radicalement de la première, si l'on reprend le raisonnement de Martin Heidegger. Pour l'écrivain, il s'agit de mettre en œuvre un processus synopsique ou synesthésique intégré à un contexte nominatif. Voilà comment Proust utilise l'intuition originaire et l'énumération-nomination en fonction du traitement esthétique d'un raisonnement sériel.

Bien que la relation poétique entre l'image et la sonorité du nom soit prioritaire, le romancier souligne de façon pénétrante que les Guermantes assassins sont charmants, intelligents, aimables. Le fait d'être assassin est le trait particulier de quelques Guermantes, mais cette caractéristique est commune à toute lignée aristocratique aussi ancienne que la série en cause. Le narrateur laisse sous-entendre que parmi cette série de meurtriers, il pourrait y avoir des tueurs en série. Il montre progressivement le rapport de coexistence qui s'établit au sein de son monde langagier entre l'universel et le particulier, le concret et l'abstrait. Il abandonne alors la désignation en ligne droite au profit de la connotation: "Les deux autres traits constitutifs de la mentalité des Guermantes étaient moins universels. " 46 Il note encore que "l'un de ces traits était la croyance que l'intelligence et aussi la bonté, la piété consistaient en choses extérieures, en connaissances" "47. Aux dires de Proust et Ockham, faut-il le rappeler, il n'y a pas d'universaux extramentaux. Rien d'étonnant alors si l'écrivain se moque des positivistes qui nient l'importance de l'intériorité. Selon lui, les positivistes ne jurent que par la construction de récits factuels qu'ils croient véridiques et qu'ils tiennent pour scientifiques. En fait, toutes leurs vérités sont sociales. Dans la psychologie de Proust, ces vérités reposent donc fondamentalement sur le snobisme et la doxa.

45. Marcel Proust, Contre Sainte-Beuve, p. 268.

46. Ibid., p. 266.

47. Ibid., p. 267. 
Le romancier étaye son propos par le biais d'une analyse des lettres et des syllabes composant le nom. Chaque lettre se pose comme un hiéroglyphe spirituel, une sorte de matière-forme. Les mots s'apparentent à des petites phrases musicales suggérant la longue phrase narrée de l'existence. C'est la traduction de la phrase du livre intérieur qui est visée. Cette longue phrase est comparable à celle de Vinteuil et de Henri Bergson. On observe ainsi que le langage mental de Proust est sémantique dans la mesure où chaque syllabe correspond à des images ou à des souvenirs. Les écarts "entre les syllabes" sont comblés par des lieux imaginaires, des domaines: "Chaque nom noble contient dans l'espace coloré de ses syllabes un château [...]." ${ }^{48}$ Le narrateur met en relief l'écart poétique du signe à l'étant lorsqu'il poursuit avec "[...] tout autour la poésie de son étang, et de son église, qui à son tour répète bien des fois le nom, avec ses armes, sur ses pierres tombales [...]" ${ }^{49}$. Il s'agit d'un art héraldique dans lequel le nom prend figure d'armoiries ou d'emblème ${ }^{50}$. Tout se passe comme si le sarcasme et la poésie se métamorphosaient en une mixture empoisonnée. De fait, Proust conjugue l'ironie du beau à la laideur des automatismes. Du même élan, il calomnie la bigoterie et le conditionnement systématique qui la sous-tend ${ }^{51}$. Il montre la supériorité poétique de l'aristocratie imaginaire qu'il met en rapport avec l'histoire de la noblesse normande: "[...] avec la vue de ce vallon dès le vIII ${ }^{\mathrm{e}}$ siècle, quand Charlemagne n'existait pas encore, quand ne s'élevaient pas les tours de la cathédrale de Chartes [...]"52. L'idéal romanesque de Proust s'éloigne manifestement des considérations esthétiques romantiques de Victor Hugo et des conceptions positivistes d'Auguste Comte.

Afin de mettre en relief les jeux homonymiques inhérents à la fonction de nomination, l'écrivain s'attarde à la correspondance du nom au signe et du signe à l'étant. Il écrit le "Nom" avec une

48. Marcel Proust, Contre Sainte-Beuve, p. 268.

49. Ibid., p. 268.

50. Voir Antoine Compagnon, La seconde main ou le travail de la citation, Paris, Seuil, 1979, p. 258-266.

51. À l'occasion, Proust s'attaque à l'idolâtrie de Ruskin dans sa traduction de Sésame et les lys et au déraillement de l'expression de Sainte-Beuve dans Contre Sainte-Beuve. Mentionnons que le romancier connaissait bien la théorie du nom masqué de Ruskin. Voir John Ruskin, Sésame et les lys, traduction et notes de Marcel Proust, édition établie par Antoine Compagnon, Verviers, Éditions Complexe, 1987, c 1906, 315 p.

52. Ibid., p. 270. 
64

majuscule alors qu'il s'y réfère comme à "cette chose antérieure à la connaissance" 53. Au moyen d'homonymes, il thématise le rapport du signe-cygne à l'étant-étang: "[...] le cri rauque et rare fait penser que s'est incarné en lui [le Nom] un des cygnes de l'étang [...]"54. Sans contredit, l'œuvre proustienne oppose le pouvoir de l'imagination aux avoirs matériels des nobles et au scientisme positiviste. Sur un ton ironique, le romancier incite le lecteur à imaginer des nobles différents de ceux qui existent dans le monde. Contrairement à ceux de ce monde, ces nobles seraient pourvus d'une sensibilité artistique: "(Peut-être les faudrait-il pas intelligents et que leur conversation n'ait trait qu'à des choses de lieu, comme ces descriptions qui ne sont évocatrices que s'il y a des images précises et pas d'abstractions.) " 55 Le narrateur confronte sa topologie de genre leibnizien à la cartographie des géographes. Il est un archéologue du savoir et un cartographe de l'imaginaire. Il récuse un matérialisme grossier au profit de la signification des lieux communs imaginaires. À l'instar de Montaigne et d'Oscar Wilde, Proust souligne le ridicule des titres de noblesse. Il raille l'emprise imaginaire de l'aristocratie sur le sens commun et le peuple. Plus encore, il remet en cause l'existence des princes: "Assurément, il n'y en eut jamais. Mais dans le seul sens imaginatif où il peut y en avoir [...]. "56 Le romancier montre que le charme des noms de la noblesse est arbitraire et qu'il opère des effets rhétoriques particuliers sur l'imagination des lecteurs et des romanciers.

Les investigations littéraires et philosophiques de Proust tendent à prouver que la fiction a autant d'emprise sur l'imagination que le réalisme en exerce sur l'intellect. C'est pourquoi l'écrivain fait l'apologie de la réalité et de la vivacité des rêves. Le conceptualisme dérivant du nominisme proustien est à l'image de la lyre d'Orphée. C'est une sorte d'"étant-étang" intarissable et métaphysique. Cet étant-étang révèle l'être et illustre la musicalité cristalline de la pensée, du jugement. Le conflit des facultés engendre une connaissance confuse qui dissimule l'être. Par exemple, si un sujet observe au loin une silhouette humaine immobile, il ne peut dire s'il s'agit d'un être humain réel ou d'un

53. Ibid., p. 270.

54. Marcel Proust, Contre Sainte-Beuve, p. 270.

55. Ibid., p. 271.

56. Ibid., p. 271. 
mannequin. Tant que le conflit des facultés persiste, la silhouette demeure virtuellement composée à moitié de bois et de chair. Ce raisonnement s'applique aussi au héros de la Recherche. Les vêtements nominaux du personnage intérieur (du moi) flottent au-dessus du concept de l'être. L'être est le fond inconscient de l'étant-étang. Il en jauge la profondeur. La prise de conscience du temps désigne directement l'être, contrairement aux mots et à la banalité du monde quotidien qui le recouvrent. Il s'agit d'une analogie entre l'être, la chose en-soi et la conscience. Un lien intellectuel se tisse entre ces entités. Récapitulons: la source nominale et le fondement du raisonnement sériel semblent primitifs, éloignés, ineffables, poétiques, dynamiques, ontologiques. L'investigation de la différence, de l'étrangeté ou du passé lointain correspond à une inversion des souvenirs qui dissémine les entrailles de l'inconscient. De cette dissémination résultent des effets de "génération et de corruption" qui affectent le raisonnement sériel $^{57}$. Ce phénomène s'éclaire si l'on considère l'érosion du temps vécu dans l'œuvre proustienne. On constate que des séries se rattachant à la noblesse de coeur ne contiennent pas de scories et sont pour ainsi dire soustraites à l'usure du temps :

Non seulement les nobles ont un nom qui nous fait rêver, mais au moins pour un grand nombre de familles, les noms des parents, des grands-parents, ainsi de suite, sont aussi de ces beaux noms, de sorte qu'aucune matière non poétique ne met d'interception dans cette greffe constante de noms colorés et pourtant transparents (Parce qu'aucune matière vile n'y adhère) [...]. ${ }^{58}$

Le narrateur insiste sur le caractère de la transparence prise au sens de ce qui transcende simultanément les liens de parenté et les apparences (trans-parent et trans-apparent). Nous pourrions supposer que ce mouvement remonte le courant de la série des étants singuliers jusqu'au temps des présocratiques. Le temps perdu, c'est l'enfance et, par analogie, l'âge poétique représente l'enfance de l'humanité 59 . La poésie du nom propre révèle l'être de la lignée ancestrale et son antériorité par rapport à d'autres lignées semblables. C'est ainsi que l'écrivain conçoit la transcendance naturelle

57. Aristote est l'auteur d'un ouvrage qui s'applique à l'atomisation des savoirs et à la logique. Voir J. Tricot, De la génération et de la corruption, Paris, J. Vrin, 1951, $171 \mathrm{p}$.

58. Marcel Proust, Contre Sainte-Beuve, p. 273-274.

59. Proust aborde cette problématique dans Jean Santeuil, op. cit., 1971. 
66

et spontanée de la poésie universelle ${ }^{60}$. Selon Proust, la transcendance est dans l'immanence puisque l'être se trouve dans le monde. À la série des noms nobles se greffe une histoire encyclopédique imaginaire des lieux, des races, etc. Par exemple, le croisement du père et de la mère, du genre et de l'espèce produit à la fois plus et moins qu'un fils ${ }^{61}$ : "Car ces beaux noms nobles ou sont sans histoire et obscurs comme une forêt, ou historiques et toujours la lumière projetée des yeux bien connus de nous de la mère éclaire toute la figure du fils. " ${ }^{2} \mathrm{La}$ relation analogique liant les traits du visage des parents à celui du fils modélise entièrement la création littéraire du romancier. Le contour abstrait du visage est l'empreinte de l'espèce et du père tandis que sa concrétion est celle de la lignée familiale et de la mère. La lumière intérieure est féminine, comme tout ce qui se situe dans l'ordre des apparences. À certains égards, la Recherche ressemble à un tableau de Braque ou de Picasso illustrant la tête d'un androgyne. Tandis que la chronologie des noms est féminine, le dogme de la durée indéterminée régit les rapports de coexistence au masculin. Ce dogme demeure obscur comme le négatif d'une photo. Le clairobscur produit par l'attraction et la répulsion qu'exerce l'inversion sexuelle se précise peu à peu en fonction d'une approche poétique statique et/ou dynamique de l'histoire.

Proust sait que "le moi est haïssable" et que, dans bien des cas, le particulier demeure banalement égoïste: "Peut-être sous ces noms-là trouverais-je quelque chose de si différent de moi qu'à la vérité cela serait presque de même matière qu'un Nom. " 63 Les noms nobles ressemblent parfois à un "cygne-signe" qui flotte au-dessus de "l'étang-étant", ce qui montre avec quelle aisance le narrateur métamorphose les Guermantes aussi bien en volatiles qu'en monuments. De fait, la noblesse de ces êtres imaginaires échappe aux descriptions réductrices et totalisantes. Les Guer-

60. Il est possible de rapprocher la thèse avicennienne de l'être précompris de façon transcendantale à toute saisie intentionnelle à la notion de "compréhension préontologique de l'être" introduite par Heidegger dans Être et temps. Alain De Libera, La querelle des universaux. De Platon à la fin du Moyen Âge, Paris, Seuil, 1996, p. 317.

61. Le genre a ici le sens premier de génération. Il possède aussi le sens de race qui en grec se dit guénos et genus en latin. Il désigne une origine. Voir Guillaume d'Ockham, Commentaire sur le livre des prédicables de Porphyre précédé du proême du commentaire sur les livres de l'art logique, p. 68-70.

62. Marcel Proust, Contre Sainte-Beuve, p. 276.

63. Ibid., p. 277. 
mantes apportent à la fois plus et moins que le rêve. Il est dans la nature de la signification du signe de dépasser la simple deixis. Les lieux Guermantes ne sont plus réels ou irréels. Ils deviennent transcendantaux :

Et si Guermantes ne déçoit pas, comme toutes les choses d'imagination quand elles sont devenues une chose réelle, c'est sans doute que ce n'est à aucun moment une chose réelle, car même quand on s'y promène, on sent que les choses qui sont là ne sont que l'enveloppe d'autres, que la réalité n'est pas ici, mais très loin, que ces choses touchées ne sont qu'une figure du Temps, et l'imagination travaille sur Guermantes vu, comme Guermantes lu, parce que toutes ces choses, ne sont encore que des mots, des mots pleins de magnifiques images et qui signifient autre chose. ${ }^{64}$

La fresque littéraire de Proust fait état de l'être à travers les noms nobles et la poésie. Pour l'écrivain, cette quête fantaisiste est synonyme du temps perdu. Or, il y a une pluralité de temps possibles. Les vieux objets incitent le lecteur à contempler la poésie du monde sensible. Leur aura rayonne même autour des noms qui les désignent: "L'instant où vivent les choses est fixé par la pensée qui les reflète. À ce moment-là, elles sont pensées, elles reçoivent leur forme. Et leur forme, immortellement, fait durer un temps au milieu des autres." ${ }^{65}$ Cette phrase révèle un genre de platonisme. Ce qu'il faut comprendre ici, c'est que la forme intemporelle correspond à la forme conceptuelle. Ce sont les chef-d'œuvres artistiques qui caractérisent la temporalité d'une forme canonique. L'œuvre détermine le genre comme une pierre qui s'intègre à une cathédrale.

La pierre angulaire de l'édifice proustien est le rire satirique. Sa plasticité rappelle l'édifice complexe de la conscience et la précieuse architecture de la Recherche. L'entre-expressions du rire anime le personnage intérieur. Il n'est donc pas étonnant que les vieilles pierres usées de Combray ou de Venise fassent sourire la grand-mère du romancier. Celle-ci représente une pierre précieuse qui s'imbrique aux écrits et aux souvenirs heureux de l'auteur. C'est parce qu'elle illustre un portrait sentimental de l'être que la grand-mère se rapproche du "temps à l'état pur". Elle incarne donc une figure de l'être-temps.

64. Ibid., p. 280.

65. Ibid., p. 281. 
68

Proust conçoit l'inversion sexuelle comme une force magnétique qui agit sur le psychisme à la manière de l'effet photoélectrique ${ }^{66}$. Contrairement à l'appareil photographique ou cinématographique, la phrase est le reflet d'une subjectivité pensante. C'est le véhicule intellectuel privilégié des impressions du narrateur ${ }^{67}$. À l'opposé de la cristallisation volontaire de l'expression et de la phrase, les noms propres évoquent une dimension poétique involontaire et ontologique. Leur signification transcende la matière tout en demeurant dans l'immanence.

En concluant Contre Sainte-Beuve, l'auteur disserte à la manière de Leibniz et d'Ockham. Lorsque son personnage intérieur examine de près une suite de tableaux ou de livres, il perçoit une chose qui n'est pas en eux. Cette chose, c'est l'universalité de la signification de l'œuvre d'art ${ }^{68}$. Proust conceptualise cet objet paradoxal et lui confère une vie de papier. Le personnage intérieur est entre-expressif et il est l'âme du moi. Il incarne les tensions émotives impliquées dans le continuum paradoxal du texte. Le moi de Proust est le centre matriciel des réalisations du possible. Ce centre est déchiré entre le particulier et l'universel. Sa logique combinatoire naturelle interagit entre deux mondes. Elle tend vers l'universalité et la création de tableaux ontico-ontologiques:

Et si entre ce tableau idéal et ce livre idéal dont chacun suffit à le rendre heureux, il trouve un lien plus haut encore, sa joie s'accroît encore. Car il meurt instantanément dans le particulier, et se remet à vivre dans le général. Il ne vit que du général, le général l'anime et le nourrit, et il meurt instantanément dans le particulier. Mais le temps qu'il vit, sa vie n'est qu'une extase et qu'une félicité. Il n'y a que lui qui devrait écrire mes livres. ${ }^{69}$

66. Voir Marcel Proust, Le temps retrouvé, p. 276-277.

67. Ibid., p. 250 et p. 261.

68. Proust n'est pas un réaliste platonicien. À la suite d'Aristote et des nominalistes, il pense que les mots et les noms sont relativement universels et dépendent des constructions sociales dont la légitimité et l'existence reposent sur une institution humaine. Quant à la poésie, elle est historiquement antérieure à l'arrière monde des idées de Platon. Elle le transcende à l'intérieur de l'âme qui ne peut être directement connue. L'âme se situe dans le domaine de la foi et des croyances. Seuls les sons vocaux et les choses ont une origine naturelle. Voir Alain De Libera, La querelle des universaux, De Platon à la fin du Moyen Âge, p. 58.

69. Marcel Proust, Contre Sainte-Beuve, p. 297. 
En somme, les beaux livres sont traduits dans une langue étrange et universelle: l'art. Le romancier explique clairement que, pour l'essentiel, sa philosophie de l'art consiste à peindre des tableaux de ce qui est: "Au fond, toute ma philosophie revient, comme toute philosophie vraie, à justifier, à reconstruire ce qui est. (En morale, en art, on ne juge plus seulement un tableau sur ses prétentions à la grande peinture et la valeur morale d'un homme sur ses discours.)" 70

\section{Le jeu de l'être-temps : pour une poétique de l'imaginaire nominal}

Les références à l'étant sont nombreuses et complexes. Elles entrent dans l'horizon déictique du possible. La signification se confond avec le référent de la description. Le signifié est un référent pur. La connotation demeure une référence partielle et possible qui a trait aux qualités de l'étant. Le nom propre caractérise toujours un étant singulier. Il multiplie aussi les étants relatifs au référent ultime: l'être. Comme l'indique Alféri, l'usage des noms communs intervient au cour de la connotation: "Ainsi, les variations au sein même de la référence, l'impropriété et les tropes, l'équivocité, la connotation ne mettent-elles pas en cause le privilège absolu des étants singuliers, isolés ou en série, comme raison d'être de tout signe, destination de tout langage. " ${ }^{71}$ Suivant la perspective philosophique d'Ockham, la référence directe ou en ligne droite prime toujours la connotation. La relativisation connotative se subordonne aux termes absolus. Sa valeur ontologique s'établit sur le jeu complexe de l'être-temps qui est le concept premier. La mémoire vient ensuite avec ses abstractions. Rappelons qu'à la suite de Duns Scot, Ockham fut le premier a développer substantiellement l'idée toute médiévale de "langage mental". Comment le langage mental peut-il être universel tout en étant toujours relatif à un utilisateur particulier? Voilà une question porteuse d'ambiguités qu'il y aurait lieu d'approfondir ultérieurement.

Proust s'applique à "reconstruire ce qui est" alors qu'il scrute la doxa au sein du discours d'autrui. Face aux snobs oiseux qui se préoccupent uniquement d'eux-mêmes, l'écrivain pratique

70. Ibid., p. 303.

71. Pierre Alféri, op. cit., p. 337. 
70

l'autodérision. En définitive, ce que l'auteur propose dans la Recherche, c'est un parcours singulier à travers les méandres d'un esprit satirique qui déraille, mais dont le style est supposé conduire la locomotive du lectorat à bon port ${ }^{72}$.

Le roman de Proust accorde suffisamment d'importance au nom pour que nous puissions concevoir plusieurs analogies avec le nominalisme d'Ockham. Si la philosophie proustienne du nom présente des traits similaires à celle d'Ockham, c'est parce que la pensée topologique du romancier est celle d'un cartographe de l'imaginaire. À ce propos, Alféri insiste sur le caractère cartographique de la pensée d'Ockham: "[...] ses motifs les plus récurrents sont la singularité des étants, la discrétion des séries, l'autonomie des intuitions, l'étanchéité des niveaux de langage, l'atomisation des connaissances, l'hétérogénéité des genres de discours." 73 Prise en un sens nominaliste, la philosophie ne peut être conçue comme un système: "De terrain, la philosophie en tant que telle n'en a pas. Elle ne se construit pas comme un système, comme une cathédrale (et c'est sans doute cela que l'histoire a le plus reproché à Ockham, en le comparant aux autres grands philosophes médiévaux, tels Thomas d'Aquin ou Duns Scot), elle se pratique dans la singularité d'un parcours." ${ }^{74}$ Le nominisme de Proust structure l'esthétique de la Recherche en exploitant le thème de la précarité existentielle. La cathédrale proustienne, si elle existe, est l'expression d'un mirage esthétique : celui de l'être-temps.

Au cour de la philosophie proustienne du nom, la pensée se communique par le biais de l'expression qu'elle module tout en travestissant le rire dans la parole. L'acte scriptural du romancier oscille entre la libération effective des affects et son simulacre. C'est un perspectivisme dont l'ultime paramètre est l'émotion. Telle est l'ambivalence de l'œuvre proustienne. Si le sens dérive à

72. Rappelons que dans Contre Sainte-Beuve, Proust se moque éperdument du style de l'académicien. Selon le romancier, Sainte-Beuve roulait des mécaniques tandis que son vieil esprit sénile déraillait de façon ponctuelle. SainteBeuve était un érudit, non un écrivain.

73. Pierre Alféri, op. cit., p. 472-473.

74. Ibid., p. 473. 
travers la Recherche, il est toujours refoulé dans les marges de l'encyclopédie. Il est contenu à l'intérieur des limites établies par une brillante réflexion philosophique qui porte sur le snobisme qu'entretiennent les mondains au fil des siècles. Il s'agit plus précisément d'un jeu complexe mettant en relief les compétences encyclopédiques de l'auteur et sa connaissance de la doxa. Ce dialogisme polyphonique, hétéroclite, bigarré et byzantin s'articule en une multitude de contrastes entre la littérature majeure et mineure. Pour Proust, il s'agit de devenir un classique et de faire comprendre au lecteur ce qu'est une ouvre universelle à la fin du XIX $^{\mathrm{e}}$ siècle.

Dans le prolongement de l'esthétique kantienne, l'œuvre proustienne se réduit à un système de croyances. L'écrivain recherche un sensus communis. Bien qu'elle soit aussi relativiste, l'entreprise esthétique du romancier est plus individualiste et noministe que celle de Kant. À la recherche du temps perdu est une somme littéraire et un roman philosophique. La dialectique interne du texte proustien est réglée sur une esthétique transcendantale de l'être-temps. Proust veut résorber toutes les contradictions du monde dans le style qu'il considère comme le vernis des maîtres. Le style ontologique que développe l'auteur vise à contourner les automatismes de la mémoire et la cristallisation du style lui-même. Le snobisme tend à élever le style au dessus de la doxa. Y parvient-t-il toujours? Voilà une des questions philosophiques que soulève la Recherche.

Bref, l'œuvre proustienne est une mise en scène de l'humaine condition. Dans cet univers romanesque baroque, le démon rococo de l'écrivain ressemble au ça de Freud. C'est-à-dire qu'il est à la fois coupable, juge et avocat. Il joue le rôle d'un penseur assis au seuil des portes de la modernité, ce nouvel enfer dantesque. Cet ange déchu réfléchit à la décadence culturelle de la modernité artistique, aux mœurs des citoyens de l'éternité et à l'inutilité de l'homme sur cette planète. Proust veut à tout prix neutraliser les préjugés sociaux que véhicule la doxa. Pour ce faire, il peint une grande fresque des mours en se référant aux classiques de la littérature, et parfois, à la satire telle qu'elle se pratiquait au Moyen Âge, ou tout au moins comme l'écrivain l'imagine à cette époque et à travers les siècles ${ }^{75}$. Selon Marcel

75. Voir Marcel Proust, Essais et articles, Paris, Gallimard, 1994, c 1971, p. 35. 
72

Proust, la satire est le fait de l'élite intellectuelle et le rire mécanique se veut un symbole d'éternité face à la mort. Le rire authentique est le sceau symbolique et onirique de l'humanité incrusté dans les profondeurs de l'inconscient. 